\title{
Association between Plasma Endocannabinoids and Appetite in Hemodialysis Patients: a Pilot Study
}

\author{
Allon N. Friedman ${ }^{1}$, Jeffrey Kim² ${ }^{2}$, Shaun Kaiser ${ }^{3}$, Theresa L. Pedersen ${ }^{4}$, and John W. \\ Newman ${ }^{4,5,6}$, Bruce A. Watkins, 6,7 \\ ${ }^{1}$ Indiana University School of Medicine, Indianapolis, IN 46202 \\ ${ }^{2}$ Genome and Biomedical Sciences Facility, University of California, Davis, Davis, CA 95616 \\ ${ }^{3}$ Texas Kidney Institute, Dallas, TX 75230 \\ ${ }^{4}$ Obesity and Metabolism Research Unit, USDA-ARS- Western Human Nutrition Research \\ Center, Davis, CA 95616 \\ ${ }^{5}$ West Coast Metabolomics Center, University of California, Davis, Davis, CA 95616 \\ ${ }^{6}$ Department of Nutrition, University of California, Davis, Davis, CA 95616 \\ ${ }^{7}$ Center on Aging, University of Connecticut Health Center, Farmington, CT
}

Corresponding author:

Allon N. Friedman, M.D.

550 University Blvd.

Suite 6100

Indianapolis, IN 46202

allfried@iu.edu

Tel: (317) 948-9419

Fax: (317) 944-4319 


\section{ABBREVIATIONS}

EC; Endocannabinoids

ECS; Endocannabinoid signaling

PUFA; Polyunsaturated fatty acids

SNAQ; Simplified Nutritional Appetite Questionnaire

FAME; fatty acid methyl esters

FAAH; fatty acid amide hydrolase

L-EA; linoleoyl ethanolamide

DH-EA; docosahexaenoylethanolamine 


\section{ABSTRACT}

Uremia-associated anorexia may be related to altered levels of long chain n-6 and n-3

polyunsaturated fatty acid (PUFA) derived circulating endocannabinoids (EC) and EC-like compounds that are known to mediate appetite. Our study's hypothesis was that such molecules are associated with appetite in patients with end-stage renal disease. A cross-sectional observational study was performed in 20 chronic hemodialysis patients ( 9 females, 11 males) and 10 healthy female controls in whom appetite was assessed using the Simplified Nutritional Appetite Questionnaire (SNAQ) and blood drawn in the fasting (and when applicable) predialysis state. Blood levels of PUFA and EC were also measured. Higher blood levels of the long chain n-6 fatty acid 20:4n6 (arachidonic acid) and lower levels of the long chain n-3 fatty acid 20:5n3 (eicosapentaenoic acid) were observed in female hemodialysis patients compared to controls. No differences were observed between male and female patients. In female study participants strong correlations between specific EC-like compounds and total SNAQ scores were noted, including with the n-6 PUFA derived linoleoyl ethanolamide (L-EA; $\rho=-0.60$, $\mathrm{P}<0.01$ ) and the n-3 PUFA derived docosahexaenoyl ethanolamide (DH-EA; $\rho=0.63, \mathrm{P}<0.01$ ).

The L-EA:DH-EA ratio was most strongly associated with the SNAQ score $(\rho=-0.74, P \leq 0.001)$, and its questions associated with appetite $(\rho=-0.69, P \leq 0.01)$ and satiety $(\rho=-0.81, P \leq 0.001)$. These findings support a link between circulating EC and appetite in hemodialysis patients.

Key words: endocannabinoids, hemodialysis, mass spectrometry, omega-3, omega-6, polyunsaturated fatty acids 


\section{INTRODUCTION}

The development of uremia from kidney failure is associated with anorexia and an increased risk of nutritional deficiencies [1-3]. Uremia may lead to loss of appetite by altering levels of circulating molecules known to mediate appetite like leptin, ghrelin, and neuropeptide Y [4]. Whether uremia also contributes to anorexia via the endocannabinoid (EC) system is unknown. Endocannabinoid signaling (ECS) is a potent mediator of food intake and influences energy metabolism through its regulation of orexigenic and anorectic molecules (including some of those mentioned above) and neural pathways [5-7]. EC mediators oversee complex crosstalk between the central and peripheral nervous system and the gut, muscle, and adipose tissue [8]. Elucidation of this process has led to new drug therapies for appetite-related disorders [9].

Of particular relevance, ECS activation via the brain cannabinoid receptor $\mathrm{CB} 1$ leads to stimulation of appetite [10]. Precursor molecules for the most investigated EC ligands of the cannabinoid receptor are derived from the polyunsaturated fatty acid (PUFA) arachidonic acid (20:4n-6), whereas EC-like ligands showing contrary and competing effects [11], are products of various saturated, monounsaturated and polyunsaturated fatty acids including the n-3 PUFA eicosapentaenoic acid (20:5n-3) and docosapentaenoic acid (22:5n-3) [10, 12].

Basic information on the physiology and blood content of EC and EC-like metabolites in patients with kidney failure is lacking. We therefore performed a pilot study in hemodialysis patients to characterize EC blood levels and examine their relationship to appetite. The study hypothesis, tested by measuring circulating levels of EC and their fatty acid precursors and comparing them to measurements of appetite [10], was that blood EC levels correlate with appetite. 


\section{METHODS AND MATERIALS}

\subsection{Participants}

Study participants were recruited from outpatient hemodialysis units affiliated with Indiana University School of Medicine, Indianapolis, IN, and healthy controls from the Indiana University-Purdue University Indianapolis (IUPUI) campus, Indianapolis, IN, in early 2012. The IUPUI campus institutional review board approved the protocol and all participants gave written informed consent after reviewing a written summary of the study plan. Inclusion criteria for the hemodialysis patients were age 18 or older and ability to provide informed consent. Exclusion criterion was hospitalization for any reason within the past 3 months.

\subsection{Appetite Questionnaire and Clinical Information}

The Simplified Nutritional Appetite Questionnaire (SNAQ) was developed to assess appetite and predict weight loss. SNAQ has been validated in healthy adults, including elderly adults and those in long-term care residences $[13,14]$ and used in the chronic kidney disease population [15]. SNAQ consists of four questions, individually scored from 1 to 5 , in order to give a range of 4-20 points, with lower scores predicting a greater risk of weight loss within six months [13]. Demographic and medical data were obtained from each participant through simple questionnaires. The SNAQ survey was administered at the time of blood draw.

\subsection{Measurement of Fatty Acids and Endocannabinoids}


A fasting blood draw was obtained immediately before a mid-week dialysis session from the dialysis tubing in each dialysis patient. Control subjects also had blood drawn in the fasting state. Blood was collected in EDTA-preserved tubes. PMSF, a fatty acid amide hydrolase (FAAH) inhibitor, was added to a set of samples to prevent breakdown of anandamide (i.e. arachidonoyl ethanolamide (A-EA)). However, results of the analysis with and without PMSF were not significantly different so the data were combined. Plasma fatty acid levels of polar lipids were measured by gas chromatographic analysis as previously described [16]. Fatty acid data reflect fatty acid methyl esters (FAME) as weight \% and the values are presented as means $\pm \mathrm{SD}$ of plasma polar fatty acids. Authentic external standards were used for the peak identification in GC output chromatograms. The sensitivity of the GC detector is at $10 \mathrm{ng} / \mathrm{peak}$ [17]. Plasma EC measurements were performed on $250 \mu \mathrm{L}$ aliquots using ultra-performance liquid chromatography-electrospray tandem mass spectrometry as previously described [18].

\subsection{Statistical analyses}

Standard descriptive statistics were used. Group differences were evaluated using 2-tailed t-tests after data normalization using imDEV v 1.4.2 [19]. Correlation between EC levels and SNAQ scores were assessed using Pearson correlations calculated in Microsoft Excel. P-values less than 0.05 were regarded as significantly different.

\section{RESULTS}

Twenty chronic hemodialysis patients and ten healthy female controls were recruited for the study. Mean age and body mass indices were not significantly different between patients and 
controls, whereas SNAQ scores were depressed in patients in a borderline significant manner $(\mathrm{P}=0.051$; Table 1). Plasma fatty acids were measured in all subjects and the principal PUFA that act as EC precursors are shown in Table 2. Plasma levels of arachidonic (20:4n-6) and eicosapentaenoic (20:5n-3) acids were higher and lower, respectively, in female hemodialysis patients compared to female controls. No difference was noted for these PUFA between male and female patients.

An array of EC were measured in the plasma of each of the twenty female hemodialysis patients and controls including the acyl ethanolamides of palmitate, stearate, oleate, linoleate, alphalinoleate, di homo-gamma-linolenate, arachidonate, docosaheptanoate, and docosahexenoate, and the 1- and 2-mono-acylglycerols of oleate, linoleate, and arachidonate (data not shown). The oleoyl ethanolamide (O-EA; $14.8 \pm 3.8$ vs. $22.6 \pm 9.7, \mathrm{P}=0.030)$ and linoleoyl ethanolamide (LEA; $6.1 \pm 2.1$ vs. $9.7 \pm 3.7, \mathrm{P}=0.016$ ) were elevated $\sim 1.5$ fold in hemodialysis patients. The relationship between EC levels and SNAQ score components were then evaluated (Table 3). The n-6 linoleic acid-derived EC linoleoyl ethanolamide (L-EA) and the n-3 docosahexaenoic acid-derived EC docosahexaenoylethanolamine (DH-EA) were negatively and positively correlated with the SNAQ score, respectively. The correlation between EC and satiety was the strongest among all the four sub-components of the SNAQ score (Table 3). Calculating the ratio of L-EA:DH-EA generated a very strong $(\rho=-0.74, \mathrm{P}<0.001)$ metabolic correlate to the SNAQ Score (Figure 1). The relationship between L-EA and DH-ES and SNAQ are presented in a Supplemental Figure. Of note, there were no differences in circulating DH-EA levels $(0.64 \pm 0.09$ vs $0.68 \pm 0.10, \mathrm{P}=0.33$ ) between the healthy females and female hemodialysis patients. 


\section{DISCUSSION}

Anorexia is a serious, well-recognized complication of uremia. The goal of our exploratory study, the first such study in patients with kidney failure, was to determine if a relationship in chronic hemodialysis patients exists between appetite and circulating blood levels of EC. We found a linear relationship between appetite and two EC-like compounds, L-EA and DH-EA, and their ratio. This appears to support our study hypothesis. This relationship showed the strongest association to the satiety component of the appetite score and is consistent with the appetite suppression previously associated with L-EA in rodents [12]. Interestingly, while elevated levels of the reported satiety factor oleoyl ethanolamide [20] were detected in female hemodialysis patients, the association with satiety was weaker $(\mathrm{P}<0.5)$ and no correlation with appetite scores was observed.

While the precise mechanisms by which EC and EC-like compounds mediate appetite are still being elucidated, it is clear that these effects occur at the hypothalamus, mesolimbic system, brainstem, and gut levels [6]. We do know that palmitoyl, oleoyl, and linoleoyl ethanolamides are poor cannabinoid receptor ligands and peroxisome proliferator activated receptor- $\alpha$ dependent appetite suppressants in rats, with the latter two molecules showing similar potencies $[12,21,22]$. In contrast, cannabinoid receptor activation is an important positive stimulator of dopaminergic reward seeking [23] and directly linked to appetite regulation [24], and DH-EA is a known cannabinoid receptor ligand [25]. In light of this information it is reasonable to suspect that circulating fatty acid ethanolamides such as L-EA and DH-EA could correlate to the appetite 
scores in the manner we have shown, and that the L-EA:DH-EA ratio could demonstrate an even stronger association with appetite.

Therefore, while this pilot study does not map out the precise mediating effects of EC in dialysis patients, it does suggest that circulating EC can reflect and perhaps influence appetite in this population. It also confirms our previous finding that blood levels of long chain n-3 PUFA are lower and long chain n-6 PUFA higher in hemodialysis patients compared to controls [26]. Such a state could result in relatively reduced production of EC-like ligands generated from long chain n-3 PUFA as compared to those from long chain n-6 PUFA, leading to an elevated L-EA:DH-EA ratio such as what we report. In light of the robust negative relationship between L-EA and appetite that we observed, this imbalance could help explain the anorexia of uremia. The strong inverse link between the L-EA:DH-EA ratio and appetite also suggests that n-3 PUFA derivedECs may antagonize the putative anorectic effects of L-EA. Therefore, strategies to manipulate EC levels by using EC agonists [10] or supplementing long-chain n-3 PUFA to increase n-3 EClike compounds (e.g. DH-EA) should be explored as an approach to improve appetite in anorectic dialysis patients.

Our pilot study has several strengths. The SNAQ screening tool has a reasonably high sensitivity $(82 \%)$ and specificity $(85 \%)$ to detect poor appetite [13]. Dialysis study participants with recent hospitalizations were excluded to avoid concomitant illnesses that could influence the SNAQ score and blood draws were uniform throughout the study. Analytic chemical measurements were performed using powerful mass spectrometry techniques to assess a broad array of known EC and EC-like compounds. While the mean BMI of our study population was in the obese 
range, the range of BMI was quite wide, thus allowing us to study appetite along a spectrum of body habitus. Future studies could examine the relationship between EC and appetite in patients who have not yet initiated dialysis at a time when the uremic milieu has not been affected by maintenance dialysis. The study also has limitations. It was modest in size and cross-sectional in design, thus preventing the assessment of causality. In addition, the analysis of EC did not include male hemodialysis patients (or a male control group) due to funding limitations.

In conclusion, our pilot study identified EC-like compounds that were significantly associated with appetite and satiety in chronic hemodialysis patients. In light of the well-established relationship between the $\mathrm{N}$-acyl ethanolamides and appetite, further research to confirm our preliminary findings in male dialysis patients, ascertain whether alterations in EC signaling play an important role in the anorexia of uremia, and determine whether PUFA supplementation can influence this role, are reasonable next steps.

\section{ACKNOWLEDGMENTS}

The study was supported by funds to BAW for the metabolomics collaborations at the University of Connecticut Health Center and Center on Aging. Additional support was provided by the USDA Intramural Projects 5306-51530-019-00D and 2032-5130-022-00D, and the West Coast Metabolomics Center Grant NIH U24 DK097154. The USDA is an equal opportunity provider and employer. The authors have no conflict of interest to report. 


\section{REFERENCES}

1. Aguilera A, Selgas R, Codoceo R, et al. Uremic anorexia: A consequence of persistently high brain serotonin levels? The tryptophan/serotonin disorder hypothesis. Peritoneal Dialysis International 2000;20(6):810-816

2. Carrero JJ, Aguilera A, Stenvinkel P, et al. Appetite disorders in uremia. Journal of Renal Nutrition 2008;18(1):107-113

3. Bergstrom J. Nutrition and Mortality in Hemodialysis. Journal of the American Society of Nephrology 1995;6(5):1329-1341

4. Bossola M, Tazza L, Luciani G. Mechanisms and treatment of anorexia in end-stage renal disease patients on hemodialysis. J Ren Nutr 2009;19(1):2-9

5. Di Marzo V, Goparaju SK, Wang L, et al. Leptin-regulated endocannabinoids are involved in maintaining food intake. Nature 2001;410(6830):822-825

6. Di Marzo V, Matias I. Endocannabinoid control of food intake and energy balance. Nature Neuroscience 2005;8(5):585-589

7. Matias I, Bisogno T, Di Marzo V. Endogenous cannabinoids in the brain and peripheral tissues: regulation of their levels and control of food intake. Int J Obes (Lond) 2006;30 Suppl $1: \mathrm{S} 7-\mathrm{S} 12$

8. Kim J, Li Y, Watkins BA. Endocannabinoid signaling and energy metabolism: a target for dietary intervention. Nutrition 2011;27(6):624-632

9. Despres JP, Golay A, Sjostrom L, et al. Effects of rimonabant on metabolic risk factors in overweight patients with dyslipidemia. N Engl J Med 2005;353(20):2121-2134

10. Watkins BA, Kim J. The endocannabinoid system: directing eating behavior and macronutrient metabolism. Frontiers in Psychology 2015;5 
11. Kim J, Carlson ME, Watkins BA. Docosahexaenoyl ethanolamide improves glucose uptake and alters endocannabinoid system gene expression in proliferating and differentiating C2C12 myoblasts. Front Physiol 2014;5:100

12. Diep TA, Madsen AN, Holst B, et al. Dietary fat decreases intestinal levels of the anorectic lipids through a fat sensor. Faseb Journal 2011;25(2):765-774

13. Wilson MM, Thomas DR, Rubenstein LZ, et al. Appetite assessment: simple appetite questionnaire predicts weight loss in community-dwelling adults and nursing home residents. Am J Clin Nutr 2005;82(5):1074-1081

14. Hanisah R, Suzana S, Lee FS. Validation of screening tools to assess appetite among geriatric patients. J Nutr Health Aging 2012;16(7):660-665

15. Salmean YA, Zello GA, Dahl WJ. Foods with added fiber improve stool frequency in individuals with chronic kidney disease with no impact on appetite or overall quality of life. BMC Res Notes 2013;6:510

16. Kim J, Carlson M, Kuchel G, et al. Dietary DHA reduces downstream endocannabinoid and inflammatory gene expression and epididymal fat mass while improving aspects of glucose use in muscle in C57BL/6J mice. International Journal of Obesity 2015; epub

17. Li Y, Watkins BA. Conjugated linoleic acids alter bone fatty acid composition and reduce ex vivo prostaglandin E-2 biosynthesis in rats fed n-6 or n-3 fatty acids. Lipids 1998;33(4):417425

18. Grapov D, Adams SH, Pedersen TL, et al. Type 2 diabetes associated changes in the plasma non-esterified fatty acids, oxylipins and endocannabinoids. PLoS One 2012;7(11):e48852

19. Grapov D, Newman JW. imDEV: a graphical user interface to R multivariate analysis tools in Microsoft Excel. Bioinformatics 2012;28(17):2288-2290 
20. Igarashi M, DiPatrizio NV, Narayanaswami V, et al. Feeding-induced oleoylethanolamide mobilization is disrupted in the gut of diet-induced obese rodents. Biochim Biophys Acta 2015;1851(9):1218-1226

21. Sarro-Ramirez A, Sanchez-Lopez D, Tejeda-Padron A, et al. Brain molecules and appetite: the case of oleoylethanolamide. Cent Nerv Syst Agents Med Chem 2013;13:88-91

22. Ezzili C, Otrubova K, Boger DL. Fatty acid amide signaling molecules. Bioorganic \& Medicinal Chemistry Letters 2010;20(20):5959-5968

23. Oleson EB, Cheer JF. A Brain on Cannabinoids: The Role of Dopamine Release in Reward Seeking. Cold Spring Harbor Perspectives in Medicine 2012;2(8)

24. Wise RA. Role of brain dopamine in food reward and reinforcement. Philosophical Transactions of the Royal Society B-Biological Sciences 2006;361(1471):1149-1158

25. Brown I, Cascio MG, Wahle KWJ, et al. Cannabinoid receptor-dependent and -independent anti-proliferative effects of omega-3 ethanolamides in androgen receptor-positive and negative prostate cancer cell lines. Carcinogenesis 2010;31(9):1584-1591

26. Friedman AN, Yu ZS, Tabbey R, et al. Low Blood Levels of Long-Chain n-3 Polyunsaturated Fatty Acids in US Hemodialysis Patients: Clinical Implications. American Journal of Nephrology 2012;36(5):451-458 
Table 1. Characteristics of the study participants

\begin{tabular}{l|c|c|c|c}
\hline & Female & \multicolumn{3}{|c}{ Hemodialysis Patients } \\
\cline { 3 - 5 } & $\begin{array}{c}\text { Controls } \\
(\mathrm{n}=10)\end{array}$ & $\begin{array}{c}\text { Female } \\
(\mathrm{n}=9)\end{array}$ & $\begin{array}{c}\text { Male } \\
(\mathrm{n}=11)\end{array}$ & $\begin{array}{c}\text { Totals } \\
(\mathrm{n}=20)\end{array}$ \\
\hline Age (yrs) & $55 \pm 5$ & $59 \pm 13$ & $52 \pm 15$ & $55 \pm 14$ \\
Diabetes (\%) & 20 & 22 & 55 & 40 \\
Time on dialysis (yrs) & & $10 \pm 11$ & $7 \pm 6$ & $8 \pm 8$ \\
Body mass index (kg/m $\left.{ }^{2}\right)$ & $31 \pm 5$ & $31 \pm 9$ & $30 \pm 10$ & $30 \pm 10$ \\
$\quad$ Range & $24-40$ & $16-44$ & $20-50$ & \\
3 month weight change $(\mathrm{kg})$ & ---- & $0.8 \pm 1.8$ & $-0.1 \pm 1.9$ & $0.4 \pm 1.9$ \\
6 month weight change $(\mathrm{kg})$ & ---- & $0.7 \pm 1.3$ & $0.4 \pm 2.9$ & $0.5 \pm 2.3$ \\
\hline SNAQ scores by question & & & & \\
$\quad$ Appetite & $3.8 \pm 1.0$ & $3.2 \pm 1.4$ & $3.9 \pm 0.8$ & $3.6 \pm 1.1$ \\
$\quad$ Satiety & $3.4 \pm 0.8$ & $2.2 \pm 1.2$ & $3.7 \pm 0.7$ & $3.1 \pm 1.2$ \\
$\quad$ Food taste & $3.9 \pm 0.7$ & $3.7 \pm 0.9$ & $4.2 \pm 0.8$ & $4.0 \pm 0.8$ \\
$\quad$ Normal intake & $3.8 \pm 0.6$ & $2.9 \pm 0.8$ & $3.5 \pm 0.8$ & $3.2 \pm 0.8$ \\
$\quad$ Total & $14.9 \pm 2.6$ & $12.0 \pm 3.4$ & $15.3 \pm 2.1$ & $13.8 \pm 3.1$ \\
\hline
\end{tabular}

Values are means \pm SD

There were no statistically significant $(\mathrm{P}<0.05)$ differences between groups 
Table 2. Plasma fatty acid composition of polar lipids of the study participants*

\begin{tabular}{|c|c|c|c|c|c|}
\hline \multirow[b]{2}{*}{ Fatty acid } & \multirow{2}{*}{$\begin{array}{l}\text { Female } \\
\text { Controls } \\
(n=10)\end{array}$} & \multicolumn{2}{|c|}{ Hemodialysis Patients } & \multirow{2}{*}{$\begin{array}{c}\text { P value } \\
\text { (female } \\
\text { controls vs. } \\
\text { patients) }\end{array}$} & \multirow{2}{*}{$\begin{array}{c}\mathrm{P} \text { value } \\
\text { (female vs. } \\
\text { male } \\
\text { patients) }\end{array}$} \\
\hline & & $\begin{array}{l}\text { Female } \\
(n=9)\end{array}$ & $\begin{array}{c}\text { Male } \\
(n=11)\end{array}$ & & \\
\hline Linoleic acid (18:2n-6) & $18.16 \pm 3.29$ & $18.40 \pm 2.28$ & $19.91 \pm 3.63$ & 0.86 & 0.29 \\
\hline Arachidonic acid (20:4n-6) & $11.47 \pm 1.71$ & $13.61 \pm 1.87$ & $13.35 \pm 2.61$ & 0.019 & 0.81 \\
\hline Eicosapentaenoic acid (20:5n-3) & $0.71 \pm 0.28$ & $0.38 \pm 0.09$ & $0.38 \pm 0.13$ & 0.005 & 0.94 \\
\hline Docosahexaenoic acid (22:6n-3) & $3.11 \pm 1.57$ & $2.68 \pm 0.78$ & $2.20 \pm 0.77$ & 0.46 & 0.19 \\
\hline Ratio of long chain $n-6 / n-3^{\dagger}$ & $3.72 \pm 1.29$ & $4.65 \pm 0.86$ & $4.93 \pm 0.66$ & 0.087 & 0.42 \\
\hline
\end{tabular}

${ }^{*}$ Values are means \pm SD of weight percentages.

${ }^{\dagger} \operatorname{LCn}-6=20: 2 n-6+20: 3 n-6+20: 4 n-6+22: 4 n-6+22: 5 n-6 ;$ LCn-3=20:5n-3+22:5n-3+22:6n-3

NB: Differences between male patients and control females for 20:4n-6 and 20:5n-3 were significant $(P \leq 0.01)$. 
Table 3. Plasma acyl ethanolamide correlations to SNAQ Scores in female subjects*

\begin{tabular}{cccccc}
\hline & Appetite & Satiety & Food Taste & Normal Intake & Total SNAQ \\
\hline P-EA & -0.04 & -0.09 & -0.03 & -0.01 & -0.04 \\
S-EA & -0.33 & $-0.63^{* *}$ & -0.29 & -0.14 & -0.41 \\
O-EA & -0.28 & $-0.50^{*}$ & -0.37 & -0.22 & -0.42 \\
L-EA & -0.44 & $-0.64^{* *}$ & -0.36 & $-0.51^{*}$ & $-0.60^{* *}$ \\
aL-EA & -0.22 & $-0.46^{*}$ & -0.29 & $-0.47^{*}$ & -0.43 \\
DGLA-EA & -0.1 & 0.01 & -0.39 & -0.18 & -0.15 \\
A-EA & -0.05 & -0.02 & 0.01 & -0.2 & -0.07 \\
D-EA & -0.36 & -0.37 & -0.24 & -0.46 & -0.43 \\
DH-EA & $0.63^{* *}$ & $0.59^{* *}$ & $0.54^{*}$ & 0.31 & $0.63^{* *}$ \\
L-EA:DH-EA & $-0.69^{* *}$ & $-0.81^{* * *}$ & $-0.49^{*}$ & -0.45 & $-0.74^{* * *}$ \\
\hline
\end{tabular}

"Values are Spearman's correlation $\rho$ values after data transformation to a more normal distribution. Significance of correlations are indicated at $p<0.001\left(^{* * * *}\right), p<0.01\left(^{* * *}\right)$, and $p<0.05$ (") using a 2-tailed assessment.

P-EA - palmitoyl ethanolamide; S-EA - stearoyl ethanolamide; O-EA, oleoyl ethanolamide; LEA, linoleoyl ethanolamide; $\alpha$ L-EA, alpha-linolenoyl ethanolamide; DGLA-EA - dihomogamma-linolenoyl ethanolamide; A-EA - arachidonoyl ethanolamide; D-EA - docosatetraenoyl ethanolamide; DH-EA - docosahexaenoyl ethanolamide 


\section{LEGEND}

Figure 1:Endocannabinoid ratio of L-EA:DH-EA to SNAQ score in female participants Correlation of composite appetite score with the L-EA:DH-EA ratio among all female subjects (healthy controls and patients, $n=19, P \leq 0.001$ ). 


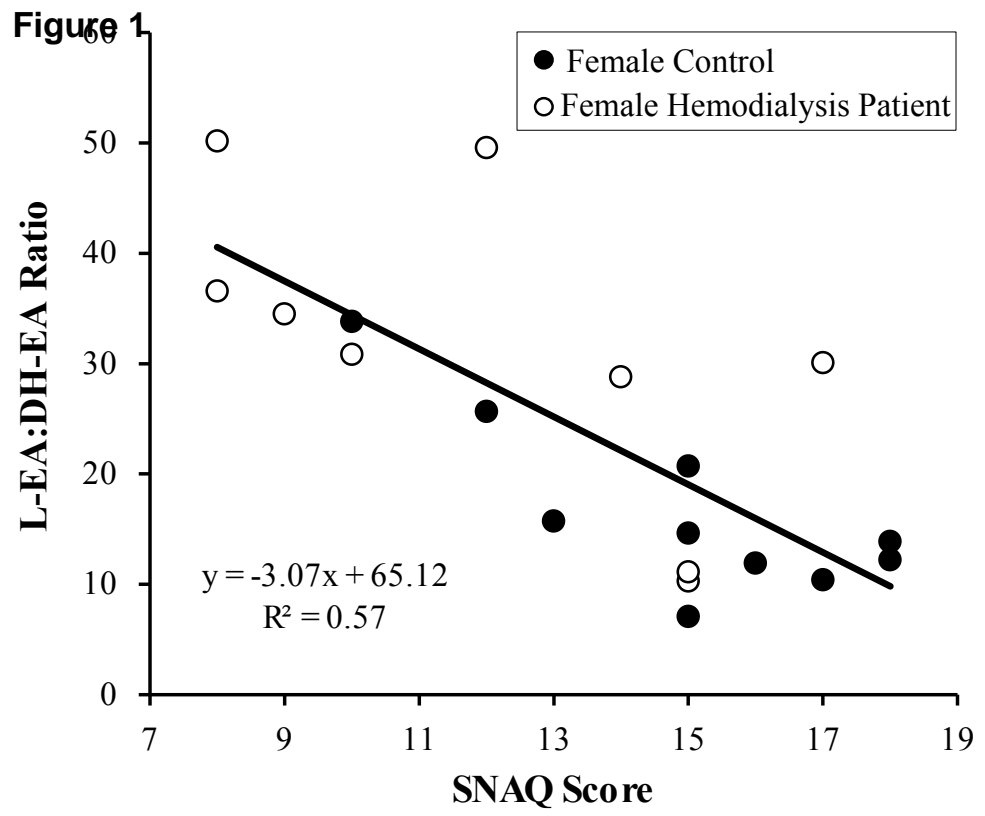

